\title{
Potential use of microwave sea surface temperatures for the estimation of ocean currents - art. no. L24608
}

\author{
J. Isern-Fontanet ${ }^{1,2}$, B. Chapron ${ }^{2}$, G. Lapeyre ${ }^{3}$ and P. Klein ${ }^{1}$
}

1 : Laboratoire de Physique des Océans, Institut Français de Recherche pour l'Exploitation de la Mer, Plouzané, France

2 : Laboratoire d'Océanographie Spatiale, Institut Français de Recherche pour l'Exploitation de la Mer, Plouzané, France

3: Laboratoire de Météorologie Dynamique, Institut Pierre-Simon Laplace, Ecole Normale Supérieure, Paris, France

\begin{abstract}
:
In this paper, we examine the emerging potential offered by satellite microwave radiometer SST measurements to complement altimeter data to quantitatively derive surface ocean currents. The proposed methodology does not follow standard sequential temporal analysis but follows the application of the Surface Quasi-Geostrophic (SQG) theory. Accordingly, under favourable environmental conditions, the implementation for this methodology is simple and robust, and most importantly, solely requires a single SST image. For the present demonstration, altimetric measurements are used to infer a necessary adjustment to match the kinetic energy level for length scales smaller than $300 \mathrm{~km}$. This helps to derive a regional effective Brunt-Väisälä frequency to produce SQG surface current estimates. As demonstrated, the results are very encouraging and strongly invite to consider the systematic use of satellite microwave radiometer measurements.
\end{abstract}

Keywords: surface quasi-geostrophy; sea surface temperature; microwave radiometers 


\title{
Potential use of microwave Sea Surface Temperatures for the estimation of ocean currents
}

\author{
J. Isern-Fontanet, ${ }^{1,2}$ B. Chapron,${ }^{2}$ G. Lapeyre ${ }^{3}$ and P. Klein ${ }^{1}$
}

In this paper, we examine the emerging potential offered by satellite microwave radiometer SST measurements to complement altimeter data to quantitatively derive surface ocean currents. The proposed methodology does not follow standard sequential temporal analysis but follows the application of the Surface Quasi-Geostrophic (SQG) theory. Accordingly, under favourable environmental conditions, the implementation for this methodology is simple and robust, and most importantly, solely requires a single SST image. For the present demonstration, altimetric measurements are used to infer a necessary adjustment to match the kinetic energy level for length scales smaller than $300 \mathrm{~km}$. This helps to derive a regional effective Brunt-Väisälä frequency to produce SQG surface current estimates. As demonstrated, the results are very encouraging and strongly invite to consider the systematic use of satellite microwave radiometer measurements.

\section{Introduction}

Global ocean surface velocities are now routinely estimated from precise altimeter range measurements. $\mathrm{Al}$ timeters can provide information about the cross-track geostrophic velocity with a relatively high along-track resolution. However, distances between tracks are generally large, and interpolation methods are required to recover both components of the surface velocity vectors [e.g. Le Traon et al., 1998]. To resolve in more details the complex space and time structure of surface currents, combined altimeters are then necessary to improve the resolution of the velocity fields [e.g. Pascual et al., 2006]. Up to 4 altimeters appear to be critical for real-time operational systems. But such a requirement may not always be met. To circumvent such a limitation, other sources of satellite data can be considered. Indeed, over the last decades, and especially for the Gulf Stream region, early space-borne optical [Strong and DeRycke, 1973] as well as recent radar high resolution observations have been reported [e.g. Chapron et al., 2005] to often illustrate striking and very well delineated complex mesoscale surface signatures related to the rich mesoscale $(30 \mathrm{~km}-200 \mathrm{~km})$ and sub-mesoscale $(<30 \mathrm{~km})$ upper ocean structures.

This wealth of high resolution information is certainly not unexpected. Klein and Hua [1990] discussed the emergence and evolution of the sea surface temperature (SST)

\footnotetext{
${ }^{1}$ Laboratoire de Physique des Océans, Ifremer, Plouzané, France

${ }^{2}$ Laboratoire d'Océanographie Spatiale, Ifremer, Plouzané, France

${ }^{3}$ Laboratoire de Météorologie Dynamique, IPSL, Paris, France
}

Copyright 2006 by the American Geophysical Union. 0094-8276/06/\$5.00 mesoscale variability triggered by a mixed-layer deepening. Such a process theoretically leads the SST to closely reproduce the characteristics of the embedding flows. As stated above, illustrations are numerous, and under favourable conditions, ocean colour and sea surface temperature (SST) data frequently reveal apparent turbulent flow patterns of very high complexities [e.g. Abraham and Bowen, 2002]. These observations and theoretical predictions thus suggests some valuable means, possibly combined with altimeter measurements, to resolve the complex surface current structures with improved resolutions [Bowen et al., 2002]. Accordingly, numerous studies have been dedicated to the problem of estimating two-dimensional ocean velocity fields from the sequences of tracer fields. Successful demonstrations dedicated to the validation and applicability of tracking features then clearly demonstrate that SST can potentially be a good proxy tracer [Emery et al., 1986; Tokmakian et al., 1990; Kelly and Strub, 1992; Bowen et al., 2002].

Among different techniques, the Maximum Cross Correlation (MCC) method is by far the most widely used. Between consecutives images, local (typically over $20 \mathrm{~km}$ window) correlations are computed to estimate shifts and the local motion. As expected, MCC shall act poorly in regions of uniform concentration of the considered scalar. As well, MCC shall have difficulties to estimate the velocity along the front of the scalar field [Zavialov et al., 2002]. Other methods have been proposed such as constrained optical flow methods to solve the heat conservation equation [e.g. Kelly, 1989; Vigan et al., 2000] or variational filtration and interpolation techniques [Afanasyev et al., 2002]. All these techniques obviously rely on the availability of cloudfree conditions and also, on very high-resolution images over short enough time periods. This latter restriction is imposed by the lack of absolute conservation of the scalar SST field. Mapping and evolution of satellite detected mesoscale features thus certainly offer valuable application to both qualitatively and quantitatively help to infer useful information. However, the stringent conditions for which these techniques can apply somehow limit geographically and seasonally the regions over which velocities can be estimated.

Low frequency microwave radiometers such as the Advanced Microwave Scanning Radiometer for EOS (AMSRE), on the other hand, are very weakly depending upon cloud conditions and can provide global SST estimates. However, their spatial resolution is of the order $20-50 \mathrm{~km}$. Such a coarser resolution compared to Advanced Very High Resolution Radiometer (AVHRR) images certainly limits the use of sequential image analysis and MCC techniques to estimate surface currents. In this paper, we thus consider a new methodology to examine the emerging potential offered by low-resolution satellite microwave radiometer SST measurements. To quantitatively derive surface currents, we consider a spatial scale analysis of the mesoscale SST variability following the application of the Surface Quasi-Geopstrophic (SQG) theory, rather than the standard sequential temporal analysis or singularity analysis [Turiel et al., 2005; IsernFontanet et al., 2006]. Applicability of such a theory to the ocean dynamics has been recently discussed [Lapeyre and Klein, 2006] and shown to be valid in numerical simulation 
of baroclinic unstable flows similar to the Antarctic Circumpolar Current. Also LaCasce and Mahadevant [2006] found a qualitative good agreement between in situ data and currents reconstructed by the SQG method. The SQG method is briefly outlined in the following section. The data are presented in section 3 , and results discussed in section 4 .

\section{Method}

In the Quasi-Geostrophic (QG) theory, the stream function $(\psi)$ of the flow can be evaluated once the potential vorticity anomaly $(Q)$ and the density anomaly at the surface $\left(\rho_{s}\right)$ are known. It consists in inverting the problem:

$$
Q=\nabla^{2} \psi+\frac{\partial}{\partial z}\left(\frac{f_{0}^{2}}{N^{2}} \frac{\partial \psi}{\partial z}\right) .
$$

with the boundary condition

$$
\left.\frac{\partial \psi}{\partial z}\right|_{s}=-\frac{g}{\rho_{0} f_{0}} \rho_{s}
$$

where $\nabla=\left(\partial_{x}, \partial_{y}\right), g$ is the gravity constant, $N$ the BruntVaisala frequency, $\rho_{o}$ a reference density and $f_{o}$ the Coriolis frequency.

If the potential vorticity anomaly $Q$ is assumed to be zero, and $N$ considered constant, the stream function at any depth can be directly evaluated. In the horizontal Fourier transform domain, the stream function simply reduces to

$$
\hat{\psi}_{s q g}(\vec{k}, z)=\frac{g}{N \rho_{0} k} \hat{\rho_{s}}(\vec{k}) \exp \left(\frac{N k z}{f_{0}}\right),
$$

where ${ }^{\wedge}$ stands for the Fourier transform, $\vec{k}$ the wave number vector and $k$ its modulus. This approximation is termed the Surface Quasi-Geostrophic (SQG) approximation [Held et al., 1995]. This corresponds to a particular situation for which the surface velocity field is now completely driven by the surface density variations. The condition of uniform PV anomaly $(Q=0)$ in (1) implies that the horizontal and vertical scales are strongly coupled as shown by (3): the smaller scales decay faster than larger ones. This hints to the possibility to compute the geostrophic velocity in the first hundreds meters of the ocean using only the surface information although in the present study we will only focus on the surface stream-function $(z=0)$.

The condition of $Q=0$ may seem unrealistic for the real ocean. However, Lapeyre and Klein [2006] recently demonstrated that baroclinic unstable flows exhibit strong correlations between ocean interior potential vorticity anomalies and surface density anomalies. The SQG concept can then be invoked, and be used considering that $N$ is replaced by an effective frequency $N_{e f f}$. This effective frequency is necessary to fix the amplitude of the SQG stream function and to implicitly take into account ocean interior dynamics not captured by the SQG theory [see Lapeyre and Klein, 2006]. To simply estimate this parameter, we recall the geostrophic estimation from the dynamic topography $(\eta)$ defined in the Fourier domain as

$$
\hat{\psi}_{g}(\vec{k})=\frac{g}{f_{0}} \hat{\eta}(\vec{k})
$$

Accordingly, the effective frequency parameter $N_{\text {eff }}$ can be evaluated to ensure the density anomaly spectrum to be congruent with the kinetic energy spectrum. Furthermore, a spectral analysis shall help to discriminate the appropriate scale range of spatial features over which the SQG methodology shall be performed.

As developed, the SQG theory assumes the near surface motion to be geostrophic, and at each scale, surface density anomalies to be in phase with dynamic topography anomalies. This is a very salient outcome of the SQG theory. The phase information is clearly essential to SQG theory to correctly position the structure at one scale with respect to structures at other scales. The SQG surface current solutions are then further distributed for each scale to provide scale-dependent vertically integrated formulations for the relationship between surface velocities and surface density gradients. In other words, while derived to describe a two-dimensional field, the SQG applies to the three dimensional system, and structures would decay away from the surface at a rate governed by their scale and $N_{\text {eff }}$. The smaller scales decay faster than larger ones. Practically, after a scale decomposition (Fourier or Wavelet transform), the SQG surface current variations can thus readily be estimated from a linear scale dependent weighting of the surface density anomaly gradients.

\section{Data}

To calculate surface currents we have used time-averages (3 days) of SST maps from version 5 of AMSR-E ocean products provided by Remote Sensing Systems (RSS) in California (USA), which have a spatial resolution of $1 / 4 \times 1 / 4$ degrees and uses correlation scales of 4 days and $100 \mathrm{~km}$ for the optimal interpolation [Yamenis and Stammer, 2004]. We have analyzed 130 maps spreading from June 5, 2002 to January 18, 2005 corresponding to a subregion of the North Atlantic ocean $(37 \mathrm{~N}-48 \mathrm{~N}, 51 \mathrm{~W}-35 \mathrm{~W})$. We have only considered those maps that had no gap (e.g. due to rain). From these maps surface density has been estimated using the UNESCO83 equation of state [UNESCO, 1983] with a constant salinity distribution fixed to $36 \mathrm{psu}$.

We have also used Delayed-Time Maps of Absolute Dynamic Topography (DT-MADT-Ref) produced by Collecte Localisation Satellites (CLS) in Toulouse (France) and distributed by AVISO, which combine the signal of two altimeters onto a $1 / 3$ degree Mercator projection grid every 7 days [SSALTO/DUACS User Handbook, 2006]. For comparison with SST data, Absolute Dynamic Topography $(\eta)$ has been interpolated to the AMSRE-E SST grid.

\section{Results}

The validity of equation (3) at the surface $(z=0)$ has been first tested by comparing the kinetic energy spectra, given by

$$
\hat{E}(k)=k^{2} \hat{\psi}^{2}
$$

associated to the stream-functions $\psi_{g}\left(\hat{E}_{g}\right)$ and $\psi_{s q g}\left(\hat{E}_{s q g}\right)$. To compute the Fourier Transform the domain is extended in each direction with mirror symetry to have it doubly periodic. Figure 1 shows a sample field of estimated surface density anomaly and Absolute Dynamic Topography corresponding to the field centered on January 5, 2005 as well as their energy spectra. For the longer wavelengths, $\hat{E}_{s q g}$ contains more energy than $\hat{E}_{g}$, which is unlikely associated directly to ocean currents but to the large-scale density forcing of the system. For shorter wavelengths $\hat{E}_{s q g}$ contains also more energy than $\hat{E}_{g}$. In this case, however, this might be due to the low-pass filters applied to altimetric data or to the correlation scales of the AMSR-E maps. In the spectral range between these two extrema, very high correlation between energy spectra of altimetry and temperature have 
been obtained. The best correlations are found, approximately, for the range between $100 \mathrm{~km}$ to $300 \mathrm{~km}$ although in some cases, such as the example shown in figure 1, there is a good coincidence also for shorter wavelengths.

Yet, the good correlation of energy spectra in the range between $100 \mathrm{~km}$ and $300 \mathrm{~km}$, is not the only factor suggesting the application of the SQG theory to reconstruct the velocity field. Indeed, the filtered fields, topography and SST, within this scale range are also found to be locally very highly correlated. In particular, the zero-crossings of the second-order derivative of these filtered fields are generally found to mostly correspond over the whole considered region. This indicates the phase information, within this scale range, to be very close for the two filtered fields. As mentioned above, this is clearly essential, as compared to spectral analysis, to correctly position the structures at different scales within the area [Armi and Flament, 1995]. Moreover, the best matches are generally found for zero-crossings of the second-order derivative fields which span the whole scale range of interest. These corresponding points shall relate to frontal areas and singularities of the first-order derivative fields from which surface velocity components are evaluated. Such findings confirm the analysis proposed by Turiel et al. [2005].

Figure 1 thus shows an example of comparisons between velocities derived from altimetry (blue) and derived from SST (red). These latter velocities are obtained by computing the inverse Fourier transform of equation (3) at the surface and setting the effective Brunt-Väisälä frequency by fitting a line to the scatter plot between altimetric velocities and SQG velocities located in regions with large thermal gradients (see figure 1). We define a non-dimensional thermal gradient as $\xi \equiv(\nabla T-\langle\nabla T\rangle) / \sigma_{\nabla T}$ where $T$ is the SST and $\sigma$ the standard deviation. Then, selecting points where $\xi>\xi_{0}$ with $\xi_{0}=2$, for the particular case of the figure, it is found that $N_{\text {eff }} f_{0}^{-1}=113$.

As seen in Figure 1, there is a good visual correlation between the two velocity fields. Therefore, there seems that the SQG method used in combination with microwave SST is able to estimate a velocity field that seems qualitatively consistent with the velocity field derived from the altimeter. Table 1 shows a more quantitative comparison obtained by computing the linear correlations $(R)$ for each component of the velocity field for the whole image and the quotient between the variance of the residual velocities and the altimetric velocities $\left(\nu_{u} \equiv \operatorname{var}\left(u_{s q g}-u_{\text {altim }}\right) / \operatorname{var}\left(u_{\text {altim }}\right)\right)$. If we focus on those regions exhibiting large thermal gradient, correlations are closer to one and residual variances are smaller. Table 1 also shows the mean values corresponding to the whole time-series.

\section{Conclusions}

In this paper, we examine the potential offered by satellite microwave radiometer SST measurements to complement altimeter data. In particular, to quantitatively derive surface ocean currents at mesoscale, we tested the application of the Surface Quasi-Geostrophic (SQG) theory. Under such a framework, we identified the SST as an active tracer proportional to $\left(-\nabla^{2}\right)^{\frac{1}{2}} \psi$, where the operator $\left(-\nabla^{2}\right)^{\frac{1}{2}}$ is defined most easily in the Fourier domain as $k$, with $\vec{k}$ a two-dimensional horizontal wavenumber. Our preliminary results show that there is indeed a robust statistical relationship of SQG type relating the SST and Sea level spectral level. Using gridded composite products, the highest correlation is found for wavelengths between $100 \mathrm{~km}$ and $300 \mathrm{~km}$ in accordance with the theoretical predictions. Most importantly, the phase information, within this scale range, is generally very close for the two filtered fields. This phase relationship leads to local high direct correlation between filtered fields.

However, strict application of SQG is apparently hampered by the use of SST fields. Indeed, the approach here proposed will be accurate only when SST would be a good proxy of the density related to mesoscale ocean structures. As understood, the SQG shall best apply to environmental conditions favouring the mixed-layer homogeneization, i.e. after strong wind events, and the reduction of air-sea surface fluxes. Nonetheless, for our demonstration using 3-day composite low-resolution radiometer data, concentrating on the largest gradients of SST fields filtered between $100 \mathrm{~km}$ and $300 \mathrm{~km}$, the SQG estimated velocities within this band are found very close to the altimeter estimates.

The results are thus very encouraging to quantitatively interpret mesoscale SST variability and strongly invite to consider the systematic use of satellite microwave radiometer measurements to complement altimeter measurements. As demonstrated, the methodology necessitates only a single image. This method can thus also complement sequential analysis, as well as to help interpretation of high resolution radar images. More importantly, the SQG applies in the context of a $3 \mathrm{D}$ system to further predict interior velocities, including vertical velocities in the upper oceanic layers. Combined with ocean colour data, future investigations can then use the simplified SQG framework to better quantify the mesoscale structure impacts on vertical tracer fluxes.

Acknowledgments. Jordi Isern-Fontanet is supported by a postdoctoral grant funded by the Spanish Ministry of Education and Science. Patrice Klein and Guillaume Lapeyre are supported by CNRS. AMSR-E data are produced by Remote Sensing Systems and sponsored by the NASA Earth Science REASoN DISCOVER Project and the AMSR-E Science Team. Data are available at www.remss.com. The altimeter products were produced by Ssalto/Duacs and distributed by Aviso, with support from CNES. Data are available at www.aviso.oceanobs.com.

\section{References}

Abraham, E., and M. Bowen (2002), Chaotic stirring by a mesoscale surface-ocean flow, Chaos, 12, 373-381.

Afanasyev, Y., A. Kostianoy, A. Zatsepin, and P. Poulain (2002), Analysis of velocity field in the eastern black sea from satellite data during the black sea '99 experiment, J. Geophys. Res., 107, doi:10.1029/2000JC000578.

Armi, L., and P. Flament (1995), Cautionary remarks on the spectral interpretation of turbulent flows, J. Geophys. Res., 90, 11,779-11,782.

Bowen, M., J. Enery, P. Wilkin, P. Tildeshey, I. Barton, and R. Knewston (2002), Extracting multilayer surface currents from sequential thermal imagery using the maximum crosscorrelation thechnique, J. Atmos. Oceanic Technol., 19, 16651676.

Chapron, B., F. Collard, and F. Ardhuin (2005), Direct measurements of ocean surface velocity from space: Interpretation and validation, J. Geophys. Res., 110, C07,008, doi:10.1029/2004JC002809.

Emery, N. J., A. C. Thomas, M. J. Collins, W. Crawford, and D. Mackas (1986), An objective method for computing advective surface velocities from sequential infrared satellite images, J. Geophys. Res., 91, 12,865-12,878.

Held, I., R. Pierrehumbert, S. Garner, and K. Swanson (1995), Surface quasi-geostrophic dynamics, J. Fluid Mech., 282, 120.

Isern-Fontanet, A., J. Turiel, García-Ladona, and J. Font (2006), Microcanonical multifractality formalism: application to the estimation of ocean surface velocities, J. Geophys. Res., submitted. 
Table 1. Correlation $(R)$ and residual variance $(\nu)$ between altimetric velocities and SQG velocities for the data centered on January 5, 2005 and the mean values for the whole timeseries $(\langle R\rangle$ and $\langle\nu\rangle)$.

\begin{tabular}{lcccccc}
\hline & $R$ & $\nu$ & $R$ & $\nu$ & $\langle R\rangle$ & $\langle\nu\rangle$ \\
\hline & Whole image & \multicolumn{4}{c}{ Large thermal gradient } \\
Zonal velocity & 0.71 & 0.49 & 0.87 & 0.24 & 0.71 & 0.50 \\
Meridional velocity & 0.67 & 0.55 & 0.90 & 0.19 & 0.69 & 0.53 \\
\hline
\end{tabular}

Kelly, K., and P. Strub (1992), Comparison of velocity estimates from advanced very high resolution radiometer in the coastal transition zone, J. Geophys. Res., 97, 9653-9668.

Kelly, K. A. (1989), An inverse model fro near-surface velocity from infrared images, Journal of Physical Oceanography, 19, 1845-1864.

Klein, P., and B. Hua (1990), The mesoscale variability of the sea surface temperature: an analytical and numerical model., $J$. Mar. Res., 48, 729-763.

LaCasce, J., and A. Mahadevant (2006), Estimating subsurface horizontal and vertical velocities from sea surface temperature, J. Mar. Res., p. submitted.

Lapeyre, G., and P. Klein (2006), Dynamics of the upper oceanic layers in terms of surface quasigeostrophy theory, J. Phys. Oceanogr., 36, 165-176.

Le Traon, P., F. Nadal, and N. Ducet (1998), An improved mapping method of multisatellite altimeter data, J. Atmos. Oceanic Technol., 15, 522-534.

Pascual, A., Y. Fauger̀e, G. Larnicol, and P. Le Traon (2006), Improved description of the ocean mesoscale variability by combining four satellite altimeters, Geophys. Res. Lett., 33, L02,611, doi:10.1029/2005GL024633.

SSALTO/DUACS User Handbook (2006), (M)SLA and (M)ADT Near-Real Time and Delayed Time Products, cLS-DOS-NT06.034 .

Strong, A., and R. DeRycke (1973), Ocean currents monitoring using a new satellite sensing technique, Science, 182, 482-484.

Tokmakian, R., P. Strub, and J. McClean-Padman (1990), Evaluation of the maximum cross-correlation method of estimating sea surface velocities from sequential satellite images, J. Atmos. Oceanic Technol., 7(6), 852-865.

Turiel, A., J. Isern-Fontanet, E. García-Ladona, and J. Font (2005), A multifractal method for the instantaneous evaluation of the stream-function in geophysical flows, Pys. Rev. Lett., 95.

UNESCO (1983), UNESCO Technical papers in marine science: Algortihms for computation of fundamental properties of seawater.

Vigan, X., C. Provost, R. Bleck, and P. Courtier (2000), Sea surface velocities from sea surface temperature images sequence 1. Method and validation using primitive equation model output, J. Geophys. Res., 105 (C08), 19,499-19,514.

Yamenis, T., and D. Stammer (2004), Time and space scales in the AMSR-E SST fields, http://www.ecco-group.org.

Zavialov, P., J. Grigorieva, O. Moller Jr., A. Kostianoy, and M. Gregoire (2002), Continuity preserving modified cross-correlation technique., J. Geophys. Res., 10\%, doi:10.1029/2001JC001.

B. Chapron, J. Isern-Fontanet, P. Klein, Département d'Océanographie Physique et Spatiale, Ifremer, BP 70, Plouzané F-29280, Plouzané, France. (bchapron@ifremer.fr, jisern@ifremer.fr, pklein@ifremer.fr)

G. Lapeyre, Laboratoire de Météorologie Dynamique, Ecole Normale Supérieure, 24 rue Lhomond, Paris F-75231BP 70, France. (glapeyre@lmd.ens.fr) 

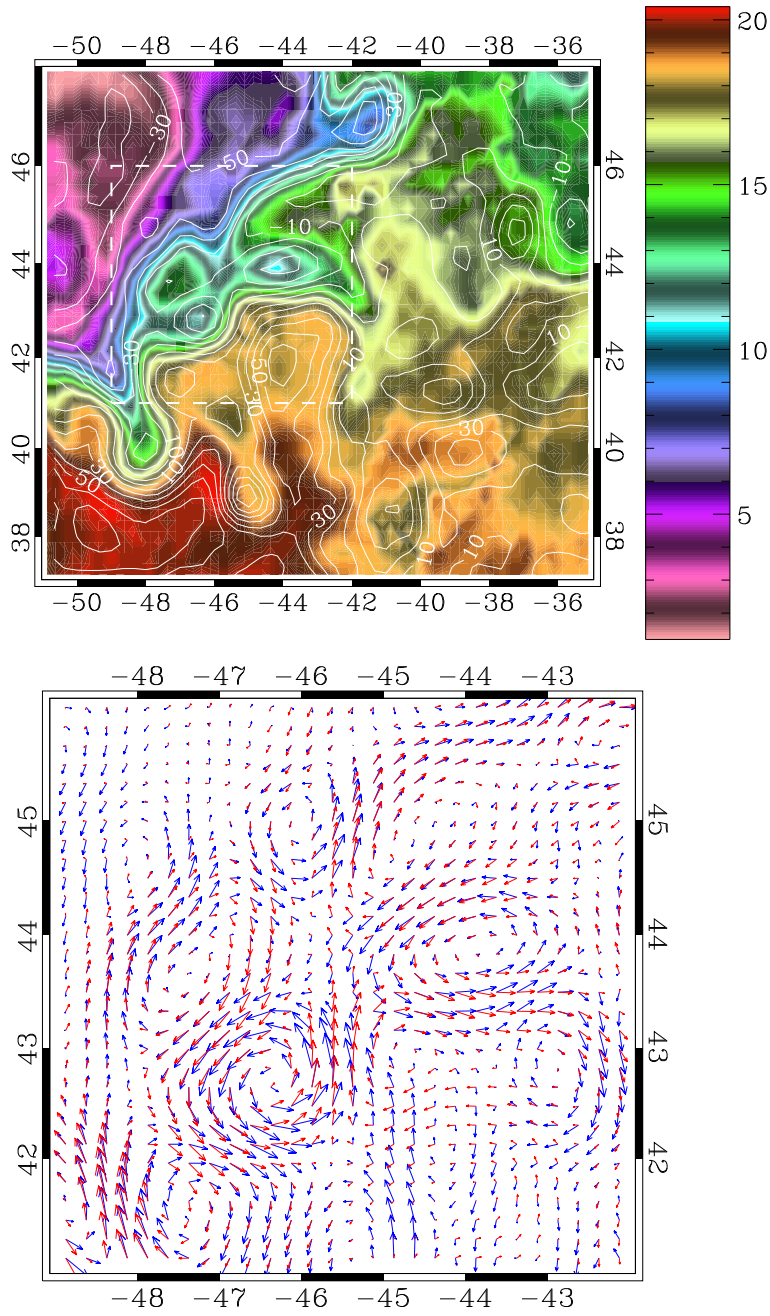

\section{0}
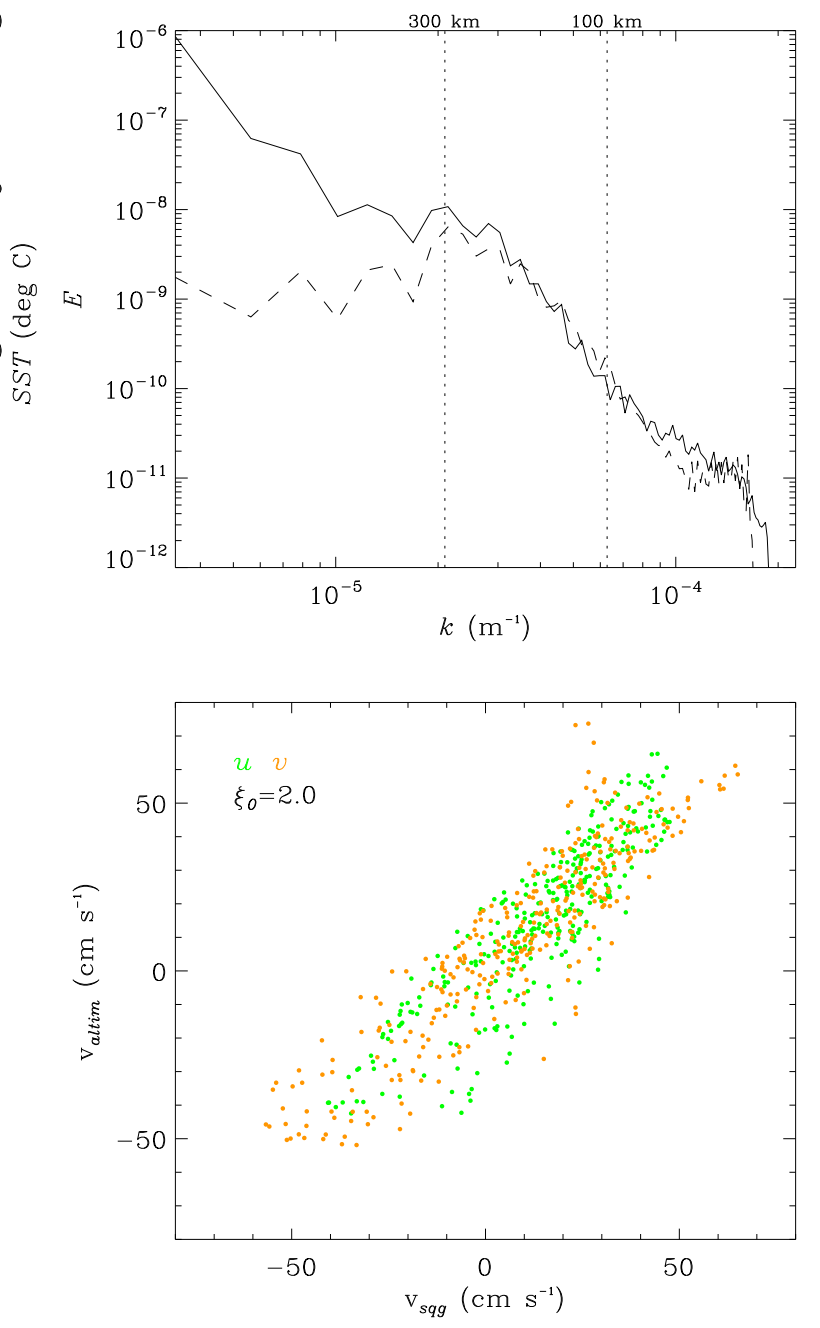

Figure 1. From left to right and from top to bottom: SST image with Absolute Dynamic Height contours superimposed (in white); energy spectra derived from SST data (solid line) and SSH (dashed line); velocities derived from microwave SST (red) and altimetry (blue) (bandfiltered retaining scales between $100 \mathrm{~km}$ and $300 \mathrm{~km}$ ) in the region delimited by the white rectangular box in the top left figure; scatter plot of the velocities located in regions with $\xi>\xi_{0}$. These plots correspond to the area limited by $37 \mathrm{~N}-48 \mathrm{~N}, 51 \mathrm{~W}-35 \mathrm{~W}$ on January $5,2005$. 